\title{
Estrategias profesionales que promueven el escepticismo en los estudiantes de contaduría pública de la ULA-NURR
}

\section{Professional strategies that promote skepticism in ULA - NURR public accounting students}

\author{
Yosman Valderrama*, Maryelin Trejo ${ }^{* *}$ y Yoximar Rangel Y** $^{* *}$
}

\section{RESUMEN}

Esta investigación tuvo por objetivo identificar las estrategias profesionales que originan el escepticismo, promovidas por parte de los docentes de Contaduría Pública de la ULA-NURR. Conducido por los postulados teóricos expuestos por:Viloria (2013), NIA 200 (IAASB, 2013a), NIA 240 (IAASB, 2013b), NIA 500 (IAASB, 2013c), Martínez et al (2010), Nelson (2009). La ruta de investigación se precisó a través de una metodología cuya tipología fue descriptiva, con diseño de campo, como población se acudió a 45 docentes del Departamento de Ciencias Económicas, Administrativas y Contables, de los cuales se seleccionaron 05 a través de un muestreo intencional, en lo concerniente a los estudiantes se consideraron los 210 inscritos en la cátedra Proyecto de tesis del semestre A-2017 en la ULA-NURR, a cuales se les aplicó un muestro probabilístico cuyo tamaño muestral estuvo representado por el 35\% de los mismos, quedando los sujetos informantes delimitados a 74 estudiantes. Como técnica se seleccionó la encuesta y como instrumento el cuestionario, cuyo análisis de datos se amparó en la triangulación de datos. Como conclusión, las estrategias profesionales que promueven el escepticismo profesional promovidas por los docentes de Contaduría Pública de la ULA - NURR, contemplan elementos esenciales de contenido, principios éticos y habilidades que permiten el desarrollo de una actitud inquisitiva en la profesión contable, esta actitud es

\footnotetext{
* Magister Scientiarum en Gerencia Empresarial. Lcdo. en Contaduría Pública. Candidato a Doctor en Ciencias Contables de la Universidad de Los Andes, y participante del Doctorado en Ciencias Gerenciales de la Universidad Rafael Belloso Chacín. Profesor Asistente. Universidad de Los Andes (ULA). Núcleo "Rafael Rangel" Trujillo. Gerente de Servicios Contables y Financieros en Valderrama Barrios \& Asociados, Trujillo - Venezuela. Correo electrónico:yosmanjose@ula.ve.

** Licenciada en Contaduría Pública, Universidad de Los Andes. Mención honorífica: Cum Laude. Investigador y Asesor Fiscal en el Libre ejercicio de la profesión. Correo electrónico:maryelin1523@ hotmail.com.

${ }^{* * * *}$ Licenciada en Contaduría Pública, Universidad de Los Andes. Mención honorífica: Cum Laude. Investigador y Asesor Fiscal en el Libre ejercicio de la profesión. Correo electrónico:yoximarrangel_06@hotmail.com.
} 
promovida a través de conversatorios, estudio de casos prácticos, revisión, análisis y evaluación de la información contable, entre otras.

Palabras clave: estrategias profesionales, escepticismo, estudiantes de Contaduría Pública.

RECIBIDO: Enero 2018 ACEPTADO: Marzo 2018

\begin{abstract}
The objective of this research was to identify the professional strategies that give rise to skepticism, promoted by the Public Accounting professors of the ULA-NURR. Conducted by the theoretical postulates exposed by: Viloria (2013), NIA 200 (IAASB, 2013a), NIA 240 (IAASB, 2013b), NIA 500 (IAASB, 2013c), Martinez et al (2010), Nelson (2009). The research route was determined through a methodology whose typology was descriptive, with field design, as population was attended 45 teachers of the Department of Economic, Administrative and Accounting Sciences, of which 05 were selected through intentional sampling, regarding the students, the 210 students enrolled in the thesis project of semester A-2017 at ULA-NURR were considered, to which a probabilistic sample was applied whose sample size was represented by $35 \%$ of them, The reporting subjects were limited to 74 students. As a technique, the survey was selected and as an instrument the questionnaire, whose data analysis was supported in the triangulation of data. In conclusion, the professional strategies that promote professional skepticism promoted by the ULA - NURR Public Accounting teachers, contemplate essential elements of content, ethical principles and skills that allow the development of an inquisitive attitude in the accounting profession, this attitude is promoted through discussions, case studies, review, analysis and evaluation of accounting information, among others.
\end{abstract}

Key words: professional strategies, skepticism, students of Public Accounting.

\title{
Introducción
}

Para el International Auditing and Assurance Standards Board (IAASB, 2016) el propósito de la auditoría financiera es expresar una opinión sobre si los estados financieros han sido preparados respecto de todo lo importante de conformidad con el marco de referencia de información financiera aplicable a la entidad, para ello, el auditor debe obtener evidencia suficiente y apropiada que le permita sustentar tal opinión a fin de aumentar el grado de confiabilidad de la 
Estrategias profesionales que promueven el escepticismo en los estudiantes de contaduría pública de la ULA-NURR

información financiera que presentan las entidades para una fecha o por un periodo determinado.

En consecuencia, el profesional contable desarrollando esta actividad profesional definida por el gremio profesional como ejercicio del contador, debe aplicar un conjunto de estrategias que le conduzcan en su labor y le permitan obtener información confiable y oportuna que reconozca la expresión de una opinión de acuerdo a la realidad económica de las entidades y vele por el interés público de esta información.

Dentro de este conjunto de estrategias profesionales, el escepticismo profesional posee un rol fundamental en el desarrollo de una auditoría de estados financieros, puesto que esta al ser ejecutada por un profesional contable independiente debe valerse de una mentalidad cuestionante sobre toda la información que se le presenta, lo que permitirá evaluar, analizar y concluir sobre la información financiera y contable de una manera objetiva, independiente e imparcial de conformidad con el requerimiento profesional y las necesidades de los mercados actuales.

En este contexto, la presente investigación tuvo como propósito identificar las estrategias profesionales que originan el escepticismo, promovidas por parte de los docentes de Contaduría Pública de la ULA-NURR, para lo cual se estructuró en un estudio dividido en su presentación en cuatro componentes básicos: 1) aspectos metodológicos; 2) fundamentos teóricos; 3) resultados de investigación; y 4) conclusiones. Este desarrollo se explana a continuación.

\section{Aspectos metodológicos}

La investigación se desarrolló siguiendo una combinación del enfoque positivista y post positivista, a razón de lo cual, se aplicó un estudio cuantitativo en el análisis de la perspectiva de los estudiantes sobre las estrategias profesionales que promueven el escepticismo, promovidas por parte de los docentes de Contaduría Pública de la ULA-NURR. Por su parte, se acudió a una orientación cualitativa que 
permitió interpretar los datos suministrados por los docentes sobre el mismo aspecto abordado a los estudiantes, todo ello con el propósito de alcanzar una triangulación de datos a fin de contrastar y confrontar las opiniones de estudiantes, docentes y fundamentos teóricos para llegar a conclusiones razonables y aportar al avance de la ciencia desde una perspectiva metodológica.

En lo que respecta al tipo de investigación, se condujo a través de un estudio descriptivo, el cual de acuerdo a Rodríguez (2005) “comprende la descripción, registro, análisis e interpretación de la naturaleza actual, composición o procesos de los fenómenos. El enfoque se hace sobre conclusiones dominantes, o sobre como una persona, grupo o cosa, se conduce o funciona en el presente" (p.24).

Como estrategia de investigación, se aplicó un diseño de campo definido por Arias (2012) como "aquella que consiste en la recolección de datos directamente de los sujetos investigados, o de la realidad donde ocurren los hechos sin manipular o controlar variable alguna" (p.31). De este modo, el trabajo fue de corte aplicativo lo que permitió comprender las estrategias profesionales que promueven el escepticismo profesional, desde la concepción práctica de los involucrados (estudiantes y docentes).

Como población se seleccionó al total de docentes adscritos al Departamento de Ciencias Económicas, Administrativas y Contables de la Universidad de Los Andes (ULA), Núcleo Rafael Rangel, Trujillo - Venezuela, que para el momento de la investigación eran cuarenta y cinco (45), quienes tienen la responsabilidad de administrar planificar y coordinar de la carrera de Licenciatura en Contaduría Pública en la ULA - Trujillo, estos se encuentran a sus vez divididos en cinco áreas de conocimiento a saber: finanzas, estadística, economía, administración y contable. Por lo cual, se aplicó un muestreo intencional siguiendo las concepciones teóricas de Scribano (2007) quien afirma que éste consiste en la selección de las unidades de la muestra en forma arbitraria, se autoseleccionan y se eligen de acuerdo a su fácil disponibilidad. 
Estrategias profesionales que promueven el escepticismo en los estudiantes de contaduría pública de la ULA-NURR

De este modo, los sujetos informantes para el ámbito profesoral estuvo integrado por los docentes del área contable que cumplieran la condición de haber impartido la materia de auditoría a los estudiantes de la carrera de Contaduría Pública en los últimos cuatro años, quedando la muestra representada por 05 docentes.

En lo que respecta a los estudiantes, la población estuvo compuesta por los educandos del décimo semestre de la carrera de Contaduría Pública de la Universidad de los Andes, Núcleo "Rafael Rangel" que cumplieron con la condición de haber inscrito Tesis de Grado en el período A-2017, considerando que éstos se encuentran culminando la carrera, por ello, fue importante conocer la perspectiva que tienen sobre el escepticismo profesional, los cuales según los datos proporcionados por la Oficina de Registros Estudiantiles ascienden a 210 estudiantes, a cuyo número se le aplicó un muestreo de tipo probabilístico considerando una selección de 35\% de la población referida (210 estudiantes), fundamentado en los postulados de Ramírez (1999) quien indica que "la mayoría de los autores coinciden que se puede tomar un aproximado del $30 \%$ de la población y se tendría una muestra con un nivel elevado de representatividad" (p.91); quedando de esta manera la muestra representada por 74 estudiantes.

Como técnica de recolección de datos, se acudió a la encuesta y como instrumento para los estudiantes un cuestionario contentivo de cuatro ítems, validados por el juicio de tres expertos en el área de conocimiento. Por otra parte, para investigar a los docentes se aplicó como instrumento el cuestionario no estructurado, y auto administrado, contenido de cuatro ítems. Fundamentado en lo expresado por García (2004) quien señala que este tipo de cuestionarios tienen preguntas que carecen de una estructura definida y de orden de aplicación rígida o precisa con lo que se genera un estilo más libre de interacción, las preguntas son abiertas y muy generales, por lo tanto, las respuestas son postcodificadas.

Finalmente, como técnica de análisis y presentación de datos se acudió a una triangulación de datos, definida por Monje (2011) como la utilización de una gran 
variedad de fuentes de datos en un estudio, esto implica comprobar la concordancia de los datos recogidos de cada de una fuentes. Lo que permitió reconocer y analizar datos desde distintos ángulos es decir, las perspectivas, de los docentes, estudiantes y la teoría para comprobarlos y contrastarlos entre sí.

\section{Fundamentación teórica}

Los auditores deben valerse de un conjunto de estrategias profesionales que le permitan cuestionar la información suministrada por su cliente, a fin de evitar influencias en su juicio profesional y la reducción de la posibilidad de concluir erróneamente, al respecto, Viloria (2013) comenta que una actitud adecuada de escepticismo profesional guía el diseño, la extensión y la oportunidad de las pruebas de auditoría, es decir, el cuestionamiento sobre la información auditada, orientará las características de la recopilación de la evidencia y sustentará el dictamen del auditor.

En este contexto, la Norma Internacional de Auditoría 200 (IAASB, 2013a) señala que el profesional escéptico debe prestar especial atención a: a) la evidencia de auditoría que contradiga otra evidencia de auditoría obtenida; b) la información que cuestione la fiabilidad de los documentos; c) las condiciones que puedan indicar un posible fraude; d) las circunstancias que sugieran la necesidad de aplicar auditoría.

De esta manera, para el auditor es fundamental aplicar procedimientos de auditoría fundamentados en poner en práctica el escéptico a fin de obtener evidencia para contribuir con la sustentación de la opinión profesional expresada en el dictamen, en otras palabras, actuar escépticamente es aplicar procedimientos de auditoría que representen el cuestionamiento de la información y vigile cualquier circunstancia contradictoria a los resultados obtenidos en la aplicación de cada prueba. Siendo así, la presente investigación desarrolla teóricamente como estrategias profesionales para aplicar el escepticismo profesional: 1) obtención de evidencia; 2) procedimientos corroborativos; 3) indicios de fraude; y finalmente, 4) identificación de necesidad de aplicar auditoría. 
Estrategias profesionales que promueven el escepticismo en los estudiantes de contaduría pública de la ULA-NURR

\section{Obtención de evidencia}

La obtención de la evidencia de auditoría es una de las estrategias que utiliza el auditor para cuestionar la información, la Norma Internacional de Auditoría 500 (IAASB, 2013c) se refiere a la evidencia como a aquella información que usa el auditor para alcanzar las conclusiones en las que se basa su opinión, incluye la información contenida en los registros contables de los que se obtiene los estados financieros, así como otra información. Siendo así, el auditor puede obtener evidencia mediante la aplicación de procedimientos, entre los cuales se mencionan los procedimientos de valoración del riesgo, pruebas de controles y procedimientos sustantivos.

Para el Public Company Accounting Oversight Board (2012) aplicar adecuadamente el escepticismo profesional es fundamental para la obtención de evidencia de auditoría suficiente, que permita determinar si las declaraciones financieras están libres de errores significativos y si en una auditoría integrada los controles internos sobre los informes financieros están operando de manera efectiva.

Por tanto, es de vital importancia la obtención de la evidencia, pues le permitirá al auditor adquirir todas aquellas pruebas que sirvan para determinar la exactitud e integridad de la información y así fundamentar su opinión, además es relevante que el profesional contable desarrolle una actitud escéptica al momento de realizar los procedimientos de auditoría.

\section{Procedimientos corroborativos}

Los procedimientos corroborativos forman parte de las estrategias de cuestionamiento, pues son necesarios para que el auditor pueda tener seguridad de la información y así emitir una opinión, en relación a esto, la Norma Internacional de Auditoría 240 (IAASB, 2013b) indica que la dirección de las entidades, con frecuencia, está en situación de privilegio para cometer fraude. Por consiguiente, el auditor, al evaluar las respuestas de la dirección a sus indagaciones con una actitud de 
escepticismo profesional, puede juzgar necesario corroborar las respuestas a sus indagaciones con otra información.

Por su parte, la Norma Internacional de Auditoría 500 (IAASB, 2013c) expresa que la corroboración de la evidencia obtenida mediante indagaciones es a menudo de especial importancia, en el caso de investigaciones sobre la intención de la dirección, la información disponible para confirmar dicha intención de la dirección puede ser limitada. Para lo cual la NIA 620 (IAASB, 2013d) en el apartado A33 recomienda los siguientes:

1) Observación del trabajo del experto del auditor;

2) Examen de los datos publicados, como informes estadísticos de fuentes reputadas y autorizadas;

3) Confirmación de personas relevantes con terceros;

4) Aplicación de procedimientos analíticos detallados; y

5) Re ejecución de cálculos.

En virtud de lo anterior, el auditor con una actitud de escepticismo profesional podrá reconocer si los encargados de la administración están expuestos a cometer fraude, esto permitirá la emisión de un juicio crítico razonable y la determinación de la necesidad de corroborar la información, en cuyo caso, la indagación puede proporcionar los datos requeridos para confirmar la evidencia obtenida.

\section{Indicios de fraude}

Los indicios de fraude que tenga el contador o en tal caso el auditor, también constituyen una actitud de duda, al respecto la Norma Internacional de Auditoría 240 (IAASB, 2013b) expone que el auditor al obtener un entendimiento de la entidad y de su entorno, incluyendo su control interno, puede identificar eventos o condiciones que indiquen un incentivo o presión para cometer fraude o que presenten una oportunidad 
Estrategias profesionales que promueven el escepticismo en los estudiantes de contaduría pública de la ULA-NURR

para cometerlo. Estos eventos o condiciones son conocidos como factores de riesgo de fraude.

Nelson (2009) expresa que el auditor deberá conducir el análisis de riesgos reconociendo la posibilidad de encontrar errores materiales debidos a fraude, esto se logrará, independientemente de la experiencia profesional con la entidad y librándose de las creencias del auditor sobre la honestidad de la gerencia y su integridad. Es decir, el escepticismo profesional requiere un cuestionamiento permanente sobre si la información y la evidencia obtenida sugieren que un error importante debido al fraude ha ocurrido.

Significa entonces, que el auditor no fundamentará su trabajo sobre la información proporcionada por la entidad considerando un grado absoluto de certeza, por tanto, se encontrará alerta ante situaciones con indicios de fraude, razonando sobre la exposición de la entidad a hechos fraudulentos, así mismo, el profesional contable considerará la exposición de la entidad ante cualquier oportunidad para realizarlo.

\section{Identificación de necesidad de aplicar auditoría}

Realizar auditorías a las entidades surge como una medida para ejercer control sobre ellas, al cerciorarse del cumpliendo con las normas que las regulan y su posible exposición a fraudes, en este sentido para Martínez et al (2010) resulta necesario introducir nuevos y mejores instrumentos de control, así como, planteamientos más rigurosos de regulación y supervisión, que coadyuven en la lucha contra la ineficacia y corrupción descubierta tras los sucesos que han afectado a la economía mundial, debido a esto surge la necesidad de aplicar auditorías como un medida de control para las entidades.

Al respecto, la Norma Internacional de Auditoría 200 (IAASB, 2013a) expresa que el objetivo de una auditoría es aumentar el grado de confianza de los usuarios en los estados financieros. Esto se logra mediante la expresión, por parte del auditor, emitiendo una opinión sobre si los estados financieros han sido preparados, en 
todos los aspectos materiales, de conformidad con un marco de información financiera aplicable.

Así pues, la realización de auditorías se torna necesaria para identificar los posibles fraudes y riesgos que se puedan presentar en las empresas, pues sirven como una medida de control para las mismas, generando confianza a los usuarios al expresar una imagen fiel en los estados financieros, para ello deben realizarse procedimientos con el fin de evaluar dichos riesgos y así evitar que estos puedan suceder.

En este contexto, Viloria (2013) expresa que la educación universitaria, puede llegar a relacionarse con el desarrollo de una actitud escéptica en el profesional, en aquellas asignaturas que se aplique el estudio de casos reales o virtuales, en los cuáles se demuestre la gestión intencionada o de omisión, por desviaciones de principios, incumplimiento de normas y hasta la manipulación de información.

De esta manera, es importante resaltar el rol que posee la universidad en el desarrollo de una actitud escéptica, pues es allí donde se proporcionan las estrategias que van a permitir mantener una mentalidad cuestionante en el ambiente laboral. Cabe agregar que, durante la formación académica los futuros profesionales deben conocer los principios y normas contables, para que de este modo sean capaces de identificar errores, desviaciones u omisiones en los mismos, siendo así, el nivel de escepticismo aumentará y se podrá descartar circunstancias que comprometan el juicio profesional.

\section{Estrategias de profesionales abordadas en la formación profesional. Los hallazgos de investigación}

Los auditores escépticos deben tener una inquietud sobre la fiabilidad de la información que recopilan, debido a ello, deben contar con estrategias de cuestionamiento para realizar una evaluación crítica de la validez de la evidencia de auditoría obtenida que contradiga o lleve a preguntarse acerca de la veracidad de los documentos presentados por la gerencia, con el objetivo de tener información 
Estrategias profesionales que promueven el escepticismo en los estudiantes de contaduría pública de la ULA-NURR

suficiente y apropiada, para poder llegar a conclusiones razonables sobre las cuales basar su opinión.

En el presente estudio abordó a los estudiantes de Contaduría Pública, así como, a los docentes de estos, a fin de identificar ¿cuáles son las estrategias de formación aplicadas para promover el escepticismo profesional en los futuros contadores público?, los resultados se explanan analíticamente a continuación.

\section{Obtención de evidencia}

Todo auditor escéptico debe obtener evidencia suficiente y adecuada, por medio de la aplicación de procedimientos que se consideren necesarios para ello, con la finalidad de tener una base razonable de la información que examina para poder expresar una opinión sobre la misma. Por consiguiente, a fin de identificar las estrategias de formación profesional que los docentes de la Carrera de Contaduría Pública de la Universidad de los Andes, Núcleo "Rafael Rangel” han aplicado en sus estudiantes, se formuló el siguiente ítem: "durante su proceso de formación los docentes del área contable le han promovido estrategias para", los resultados desde la perspectiva de los estudiantes se presentan en la tabla 1.

Tabla 1. Obtención de evidencia desde la perspectiva de los estudiantes

\begin{tabular}{|l|c|c|}
\hline \multicolumn{1}{|c|}{ Respuestas } & $\begin{array}{c}\text { Frecuencia } \\
\text { Absoluta }\end{array}$ & $\begin{array}{c}\text { Frecuencia } \\
\text { Relativa }\end{array}$ \\
\hline Obtener información en la cual basar la opinión & 45 & $60,8 \%$ \\
\hline Utilizar procedimiento de valoración de riesgo & 40 & $54,1 \%$ \\
\hline Aplicar pruebas de controles & 42 & $56,8 \%$ \\
\hline Aplicar pruebas para detectar errores en los estados financieros & 44 & $59,5 \%$ \\
\hline $\begin{array}{l}\text { Emplear el escepticismo profesional para la obtención de suficiente } \\
\text { evidencia }\end{array}$ & 43 & $58,1 \%$ \\
\hline $\begin{array}{l}\text { No se han promovido estrategias de cuestionamiento en cuanto a la } \\
\text { obtención de evidencia }\end{array}$ & 4 & $5,4 \%$ \\
\hline Otra. Indique & 0 & $0 \%$ \\
\hline
\end{tabular}

Fuente: Instrumento aplicado a los estudiantes. 
En la tabla anterior se muestra los hallazgos del ítem 1, en el cual desde el enfoque de los estudiantes, las estrategias de formación profesional que los docentes aplican para promover las técnicas de cuestionamiento en lo que concierne a la obtención de evidencia, fueron abordadas en su mayoría, basándose en la obtención de información en la cual basar la opinión, la aplicación de pruebas para detectar errores en los estados financieros, el empleo del escepticismo profesional para la obtención de suficiente evidencia, la aplicación de pruebas de controles, así como, la utilización de procedimientos de valoración de riesgo.

En relación a lo antes expuesto, dichas estrategias podrían otorgarle a los futuros profesionales los mecanismos esenciales para que desarrollen una actitud adecuada de cuestionamiento al realizar su trabajo en el campo laboral puesto que, deben obtener evidencia necesaria para juzgar si la información es verdadera y confiable y de esta manera poder sustentar su dictamen.

No obstante, cabe resaltar que algunos estudiantes en un número no representativo indicaron que no se están aplicando estrategias para la obtención de evidencia. Por tanto, los docentes deben incrementar el fomento de las técnicas para la obtención de las mismas, por ser herramientas necesarias en todo profesional escéptico, cuyas competencias permitirán desarrollar una mente inquisitiva acerca de las evidencias recopiladas, mantenerse alerta e investigar para formarse una opinión que minimice frente a los posibles errores materiales.

Por otro lado, para examinar la perspectiva de los docentes en cuanto a las estrategias que aplican para impartir conocimientos de obtención de evidencia, se les empleó el ítem "qué estrategias aplica usted como docente para promover en sus estudiantes estrategias de cuestionamiento como la obtención de evidencia", cuyos datos recolectados de presenta en la matriz de análisis 1 que se muestra a continuación. 
Estrategias profesionales que promueven el escepticismo en los estudiantes de contaduría pública de la ULA-NURR

Matriz de Análisis 1. Obtención de evidencia desde la perspectiva de los docentes
\begin{tabular}{|l|l|}
\hline Docente 1 & $\begin{array}{l}\text { Desarrollo de programas de casos y aplicaciones prácticas basadas en experiencias y } \\
\text { trabajos exitosos de colegas en cada área. }\end{array}$ \\
\hline Docente 2 & $\begin{array}{l}\text { Revisión rigurosa y exhaustiva de la información contable tributaria con una perspectiva } \\
\text { crítica, argumentativa e interpretativa de lo que se presenta teniendo presente que ésta debe } \\
\text { ser útil para la toma de decisiones asertivas. }\end{array}$ \\
\hline Docente 3 & Casos prácticos. \\
\hline Docente 4 & $\begin{array}{l}\text { Se resalta la actitud independiente para evaluar las evidencias. Se recalca la importancia de } \\
\text { la comprobación, la indagación, la evaluación de las evidencias para poder llegar a } \\
\text { conclusiones. la principal estrategia es el análisis y estudio de casos. }\end{array}$ \\
\hline Docente 5 & $\begin{array}{l}\text { Analizar y disertar la evidencia obtenida tanto individual como grupal. Interactuar los } \\
\text { distintos grupos conformados en el aula de clase, de manera que se refuten mutuamente las } \\
\text { distintas evidencias que tenga cada grupo. }\end{array}$ \\
\hline
\end{tabular}

Fuente: Instrumento aplicado a los docentes.

El instrumento aplicado a los docentes refleja las estrategias que estos utilizan para promover la obtención de evidencia, dentro de las que se resaltan, el desarrollo de programas de casos y aplicaciones prácticas, la revisión rigurosa y exhaustiva de la información contable tributaria con una perspectiva crítica, el análisis y estudio de casos, así como, el razonamiento tanto individual como grupal de las evidencias obtenidas.

En este sentido, es pertinente resaltar que las estrategias aplicadas por los docentes para incentivar el desarrollo de obtención de evidencia ayudan a la formación de los estudiantes, puesto que crean profesionales críticos, hábiles y con una mente cuestionante que entienden que su trabajo se basa en la evidencia de auditoría apropiada. Aunque, es significativo decir que los docentes deben profundizar dichas estrategias debido a que, representan una ventaja para los auditores al brindarles las destrezas esenciales para desarrollar una competencia de investigación.

\section{Procedimientos corroborativos}

Los contadores y auditores necesitan confirmar la información sobre la que posteriormente se emitirá una opinión, pues existen datos de contabilidad que por sí solos no pueden considerarse evidencia suficiente, por lo tanto, se hace necesario la comprobación de la corrección y exactitud de la información contable, para ello los 
profesionales se valen de procedimientos corroborativos, tales como, la investigación, inspección, observación, examen físico, entre otros, pues permiten justificar las conclusiones expuestas en el dictamen.

En este sentido, para identificar las estrategias de formación profesional que los docentes de la carrera de Contaduría Pública de la Universidad de los Andes, Núcleo "Rafael Rangel" han aplicado en los estudiantes en referencia a los procedimientos corroborativos, se aplicó el siguiente ítem "con respecto a la confirmación de información, en su formación los docentes del área contable lo han estimulado a", los resultados desde la perspectiva de los estudiantes se presentan en la tabla 2 que se muestra a continuación.

\section{Tabla 2. Procedimientos corroborativos desde la perspectiva de los estudiantes}

\begin{tabular}{|l|c|c|}
\hline \multicolumn{1}{|c|}{ Respuestas } & $\begin{array}{c}\text { Frecuencia } \\
\text { Absoluta }\end{array}$ & $\begin{array}{c}\text { Frecuencia } \\
\text { Relativa }\end{array}$ \\
\hline $\begin{array}{l}\text { Evaluar las respuestas a sus indagaciones corroborándolas con otra } \\
\text { información. }\end{array}$ & 35 & $47,3 \%$ \\
\hline $\begin{array}{l}\text { Corroborar la evidencia obtenida mediante indagaciones sobre la } \\
\text { intención que tiene el personal de las entidades. }\end{array}$ & 42 & $56,8 \%$ \\
\hline Hacer uso del juicio crítico para tomar decisiones. & 47 & $63,5 \%$ \\
\hline $\begin{array}{l}\text { No se han promovido estrategias para cuestionar los } \\
\text { procedimientos corroborativos. }\end{array}$ & 5 & $6,8 \%$ \\
\hline Otra. Indique. & 0 & $0 \%$ \\
\hline
\end{tabular}

Fuente: Instrumento aplicado a los estudiantes.

En la tabla anterior se reflejan los hallazgos del ítem 2, pues desde la óptica de los estudiantes, entre las estrategias de formación profesional que los docentes han aplicado para promover técnicas de cuestionamiento relativas a procedimientos corroborativos sobre la información contable, se pueden mencionar, el uso del juicio crítico para tomar decisiones, corroborar la evidencia obtenida mediante indagaciones sobre la intención que tiene el personal de las entidades y evaluar las respuestas a sus indagaciones corroborándolas con otra información, lo cual puede evidenciar que a los estudiantes se les aplicó estrategias de formación profesional que ayudan a estimular la puesta en práctica de procedimientos corroborativos. Aunque, cabe agregar que un 261 
Estrategias profesionales que promueven el escepticismo en los estudiantes de contaduría pública de la ULA-NURR

porcentaje no representativo de los sujetos informantes indicó que no les han promovido dichas estrategias durante su proceso de formación.

Ahora bien, al analizar los hallazgos obtenidos se percibe que a los estudiantes en su mayoría se les han fomentado estrategias de formación primordiales para el desarrollo laboral, hacer uso del juicio crítico es relevante si se toma en cuenta la validez que se quiere obtener de la evidencia, en la medida en que se efectúe diversos grados de inspección, análisis y fiscalización en cada caso particular. De la misma manera, es imprescindible comprobar y evaluar la información contable con respuestas obtenidas del personal directivo, con el fin de conocer la verdadera intención de éstos, puesto que valiéndose de su condición puede manipular información.

Aun cuando se han aplicado dichas estrategias, es fundamental que continúe la promoción de técnicas para comprobar la información contable que se utilizará como evidencia que respalda una opinión. Igualmente, al tener en cuenta los procedimientos corroborativos, incrementa de alguna $\mathrm{u}$ otra manera, la actitud escéptica que debe poseer todo profesional contable.

Por otro lado, para conocer la perspectiva de los docentes, en cuanto a las estrategias de formación profesional que aplican para promover el desarrollo de procedimientos corroborativos, se implementó el siguiente ítem "qué estrategias aplica usted como docente para promover en sus estudiantes estrategias de cuestionamiento como procedimientos corroborativos", cuyos datos recolectados de presenta en la matriz de análisis 2.

\section{Matriz de Análisis 2. Procedimientos corroborativos desde la perspectiva de los} docentes

\begin{tabular}{|l|l|}
\hline Docente 1 & $\begin{array}{l}\text { Desarrollo de programas de casos y aplicaciones prácticas basadas en experiencias y } \\
\text { trabajos exitosos de colegas en cada área. }\end{array}$ \\
\hline Docente 2 & $\begin{array}{l}\text { Revisión rigurosa y exhaustiva de la información contable tributaria con una perspectiva } \\
\text { crítica, argumentativa e interpretativa de lo que se presenta teniendo presente que ésta debe } \\
\text { ser útil para la toma de decisiones asertivas. }\end{array}$ \\
\hline Docente 3 & Casos prácticos. \\
\hline Docente 4 & La correcta aplicación de la NIA en los procedimientos corroborativos la correcta aplicación \\
\hline
\end{tabular}




\begin{tabular}{|l|l|}
\hline & $\begin{array}{l}\text { de los procedimientos de auditoria. En este sentido la aplicación práctica es de suma } \\
\text { importancia, así como el análisis de la información. Particularmente trabajo con prácticas } \\
\text { simulativas y análisis de la información. }\end{array}$ \\
\hline Docente 5 & $\begin{array}{l}\text { Consultar, analizar y disertar sobre los riesgos de la auditoría en casos de estudio prácticos, } \\
\text { esto permitirá planeación e implementación de respuestas apropiadas a dichos riesgos, tal } \\
\text { como lo señala la norma. }\end{array}$ \\
\hline
\end{tabular}

Fuente: Instrumento aplicado a los docentes.

Una vez obtenidas las opiniones de los docentes, por medio del instrumento empleado, se puede comprobar que los mismos han implementado en su programación académica la aplicación de estrategias de formación basadas en el desarrollo de técnicas de cuestionamiento relativas a los procedimientos corroborativos.

Entre las estrategias utilizadas se pueden distinguir, el desarrollo de programas de casos y aplicaciones prácticas, la revisión rigurosa y exhaustiva de la información contable tributaria con una perspectiva crítica, argumentativa e interpretativa de lo que se presenta considerando que ésta debe ser útil para la toma de decisiones asertivas, el análisis de la información con respecto a la correcta aplicación de la NIA en los procedimientos corroborativos y procedimientos de auditoría, casos de estudio prácticos que consistan en consultar, analizar y disertar sobre los riesgos de la auditoría, esto permitirá la planeación e implementación de respuestas apropiadas a dichos riesgos.

Resulta oportuno decir que, las estrategias implementadas por los docentes son de gran utilidad para los estudiantes, ya que les proporcionan métodos y técnicas sobre cómo corroborar la información que será utilizada para emitir conclusiones razonables acerca de la situación financiera de la empresa, de la misma manera, el fomento de tales estrategias coadyuvan al desarrollo de la mentalidad cuestionante de los futuros profesionales, la cual es imprescindible para realizar una labor de calidad. 
Estrategias profesionales que promueven el escepticismo en los estudiantes de contaduría pública de la ULA-NURR

\section{Indicios de fraudes}

El auditor escéptico debe desarrollar una actitud inquisitiva, con el fin de estar alerta a aquellas situaciones que pudieran indicar que se están presentando errores debido a fraude o a error, por ello se debe estar atento a la información que las entidades proporcionen. En este sentido, con el propósito de identificar las estrategias de formación profesional que los docentes de la carrera de Contaduría Pública de la Universidad de los Andes, Núcleo "Rafael Rangel” han aplicado en sus estudiantes, se abordó a los encuestados con el siguiente ítem: "durante su proceso de formación los docentes del área contable le han proporcionado las herramientas para", los resultados desde la perspectiva de los estudiantes se presentan en la tabla 3.

Tabla 3. Indicios de fraudes desde la perspectiva de los estudiantes

\begin{tabular}{|l|c|c|}
\hline \multicolumn{1}{|c|}{ Respuestas } & $\begin{array}{c}\text { Frecuencia } \\
\text { Absoluta }\end{array}$ & $\begin{array}{c}\text { Frecuencia } \\
\text { Relativa }\end{array}$ \\
\hline $\begin{array}{l}\text { Identificar condiciones que indiquen un incentivo para cometer } \\
\text { fraude. }\end{array}$ & 31 & $41,9 \%$ \\
\hline Identificar condiciones que indiquen presión para cometer fraude. & 13 & $17,6 \%$ \\
\hline $\begin{array}{l}\text { Reconocer la posibilidad que un error importante debido al fraude } \\
\text { podría estar presente. }\end{array}$ & 40 & $54,1 \%$ \\
\hline $\begin{array}{l}\text { Cuestionar permanentemente la información manteniendo el } \\
\text { escepticismo profesional. }\end{array}$ & 46 & $62,2 \%$ \\
\hline $\begin{array}{l}\text { No se han promovido estrategias de cuestionamiento en cuanto a } \\
\text { indicios de fraude. }\end{array}$ & 7 & $9,5 \%$ \\
\hline Otra. Indique. & 0 & $0 \%$ \\
\hline
\end{tabular}

Fuente: Instrumento aplicado a los estudiantes.

En la tabla anterior se evidencian los resultados del ítem 3, en el cual desde la óptica de los estudiantes las estrategias de formación profesional que han aplicado los docentes en su proceso de profesionalización resaltan, el cuestionamiento permanente de la información, el reconocer la posibilidad de que un error importante, debido a fraude podría estar presente y la identificación de condiciones que indiquen un incentivo para cometer fraude. No obstante, la identificación de condiciones que indiquen presión para cometer fraude, fue la estrategia que se aplicó en menor 
proporción, resaltando que algunos estudiantes señalaron en un mínimo porcentaje que no se están aplicando estrategias en lo que concierne a los indicios de fraudes.

Los resultados del estudio, evaluados desde el ámbito del cuestionamiento permanente de la información, evidencian que el reconocer la posibilidad que un error importante debido a fraude podría estar presente y la identificación de condiciones que indiquen un incentivo para cometerlo, podrían representar un apoyo para los estudiantes de Contaduría Pública, puesto que se direcciona a los mismos a evaluar los hechos manteniendo una actitud crítica, reconociendo que es posible que existan incorreciones materiales debido a fraude.

Sin embargo, es necesario que se haga énfasis en la promoción de la identificación de condiciones que indiquen presión para cometer fraude y de las estrategias de indicios de fraudes en general, ya que por medio de ello el futuro profesional contará con los conocimientos esenciales para identificar aquellas situaciones fraudulentas que se presenten en las entidades, para ello es significativo conocer los controles de las mismas desarrollando una mentalidad de duda.

Ahora bien, para indagar sobre la perspectiva que tienen los docentes en cuanto a la aplicación de estrategias para promover la identificación de los indicios de fraudes, se formuló el siguiente ítem: "qué estrategias aplica usted como docente para promover en sus estudiantes estrategias de cuestionamiento como indicios de fraudes", cuyos datos recolectados de presenta en la matriz de análisis 3.

\section{Matriz de Análisis 3. Indicios de fraude desde la perspectiva de los docentes}

\begin{tabular}{|l|l|}
\hline Docente 1 & La revisión de la normativa, revisión de casos y experiencias en el área. \\
\hline Docente 2 & $\begin{array}{l}\text { Revisión rigurosa y exhaustiva de la información contable tributaria con una visión analítica, } \\
\text { argumentativa de las posibles desviaciones que se puedan presentar contrastando la } \\
\text { información presentado con las Normativas establecidas a tal fin, con el propósito de detectar } \\
\text { fraudes o errores que induzcan a tomar decisiones poco asertivas con un nivel elevado de } \\
\text { riesgo. }\end{array}$ \\
\hline Docente 3 & Casos prácticos. \\
\hline Docente 4 & $\begin{array}{l}\text { Cuando hay incongruencias de las fuentes de información en las evidencias, cuando no hay } \\
\text { evidencias suficientes. Les enseño a tener cuidado con la información que suministra la } \\
\text { administración, mantener reserva de los hallazgo, hasta el momento de ser presentados. } \\
\text { Utilizo el estudio de casos, así como la exposición de grupos para reforzar los conocimientos. }\end{array}$ \\
\hline
\end{tabular}


Estrategias profesionales que promueven el escepticismo en los estudiantes de contaduría pública de la ULA-NURR

\begin{tabular}{|l|l|}
\hline Docente 5 & $\begin{array}{l}\text { Conocer, analizar y evaluar la organización bajo el enfoque sistémico (La parte está en el } \\
\text { todo y el todo está en la parte). Se conocerá la entrada, proceso, salida, ambiente interno y } \\
\text { externo que posee la organización, con un análisis, estudio y conocimiento de su proceso } \\
\text { administrativo. Todo esto es aplicado a casos prácticos nacionales e internacionales. }\end{array}$ \\
\hline
\end{tabular}

Fuente: Instrumento aplicado a los docentes.

Por medio del instrumento aplicado a los docentes se puede apreciar las estrategias que utilizan para promover la identificación de los indicios de fraude, destacando entre ellas, la revisión de la normativa, revisión de casos y experiencias en el área, la revisión rigurosa y exhaustiva de la información contable, la aplicación de casos prácticos, así como, el conocimiento, análisis y evaluación de la organización bajo el enfoque sistémico.

Por tanto, debe señalarse que las estrategias aplicadas por los docentes para promover la búsqueda de indicios de fraudes proporcionan herramientas esenciales enmarcadas en la investigación y el conocimiento de situaciones irregulares manteniendo una actitud escéptica, permitiendo la conformación de evidencia necesaria para proporcionar una seguridad razonable al momento de emitir un dictamen. Siendo así, es recomendable en los docentes despertar una actitud cuestionante en los estudiantes con sus vinculaciones éticas y responsabilidad social.

\section{Identificación de necesidad de aplicar auditoría}

Aplicar auditoría surge como una estrategia para asegurarse por medio de la opinión del auditor que las entidades han preparado y presentado los estados financieros de conformidad con el marco de referencia que debe ser aplicado de acuerdo a las características de la entidad, permitiendo tomar control sobre las mismas acerca de su adecuado funcionamiento, pudiéndose identificar los riesgos, fallas y errores cometidos, para así tomar medidas que contribuyan a retomar el rumbo correcto de la misma.

En este sentido, para identificar las estrategias de formación profesional que los docentes de la Carrera de Contaduría Pública de la Universidad de los Andes, Núcleo 
"Rafael Rangel”, han aplicado en sus estudiantes en relación a identificar la necesidad de aplicar auditoría, se empleó el siguiente ítem “durante su proceso de formación los docentes del área contable le han fomentado estrategias para", los resultados desde la perspectiva de los estudiantes se presentan en la tabla 4.

\section{Tabla 4. Identificación de necesidad de aplicar auditoría desde la perspectiva de los estudiantes}

\begin{tabular}{|l|c|c|}
\hline \multicolumn{1}{|c|}{ Respuestas } & $\begin{array}{c}\text { Frecuencia } \\
\text { Absoluta }\end{array}$ & $\begin{array}{c}\text { Frecuencia } \\
\text { Relativa }\end{array}$ \\
\hline $\begin{array}{l}\text { Identificar la necesidad de aplicar auditorías como una medida de } \\
\text { control. }\end{array}$ & 60 & $81,1 \%$ \\
\hline $\begin{array}{l}\text { Aumentar el grado de confianza de los usuarios en los estados } \\
\text { financieros. }\end{array}$ & 50 & $60,5 \%$ \\
\hline $\begin{array}{l}\text { Emitir una opinión sobre si los estados financieros han sido } \\
\text { preparados en todos los aspectos materiales de conformidad con un } \\
\text { marco de información financiera aplicable. }\end{array}$ & 0 & $0 \%$ \\
\hline $\begin{array}{l}\text { No se han promovido estrategias de cuestionamiento en cuanto a la } \\
\text { identificación de la necesidad de aplicar auditoría. }\end{array}$ & 0 & $0 \%$ \\
\hline Otra. Indique. & & $0 \%$ \\
\hline
\end{tabular}

Fuente: Instrumento aplicado a los estudiantes.

En la tabla anterior se muestran los hallazgos correspondientes al ítem 4, en el cual desde la perspectiva de los estudiantes, las estrategias de formación profesional que han aplicado los docentes con respecto a identificar la necesidad de aplicar auditoría, destacan identificar la necesidad de aplicar auditorías como una medida de control, así como, emitir una opinión sobre si los estados financieros han sido preparados en todos los aspectos materiales de conformidad con un marco de información financiera aplicable. Sin embargo, aumentar el grado de confianza de los usuarios en los estados financieros, fue la estrategia que se aplicó con menor énfasis.

Estos resultados evidencian una fortaleza para el desempeño laboral del futuro profesional contable puesto que le permitirá identificar cuándo se debe realizar una auditoría a los estados financieros, así como, el momento en que es requerido expresar una opinión sobre la razonabilidad de la información contable y el cumplimiento de las normas relativas a ello. 
Estrategias profesionales que promueven el escepticismo en los estudiantes de contaduría pública de la ULA-NURR

No obstante, se requiere profundizar en la aplicación de estrategias para incentivar el trabajo de auditoría a fin de aumentar el grado de confianza de los usuarios en cuanto a los estados financieros, lo cual se logrará manteniendo el escepticismo profesional, lo que permitiría ejecutar auditorías objetivas donde se refleje la exactitud y veracidad de los registros mostrados por la entidad.

Por otra parte, para examinar la perspectiva de los docentes en cuanto a las estrategias que están aplicando para identificar la necesidad de aplicar auditoría, se formuló el siguiente ítem: "qué estrategias aplica usted como docente para promover en sus estudiantes estrategias de cuestionamiento como la identificación de necesidad de aplicar auditoría", cuyos datos recolectados de presenta en la matriz de análisis 4 .

Matriz de análisis 4. Identificación de necesidad de aplicar auditoría desde la
perspectiva de los docentes
\begin{tabular}{|l|l|}
\hline Docente 1 & $\begin{array}{l}\text { Desarrollo de elementos que permitan reconocer la importancia y aplicabilidad de la } \\
\text { Auditoria en cualquier circunstancia. }\end{array}$ \\
\hline Docente 2 & $\begin{array}{l}\text { Revisión, análisis y evaluación de los sistemas de información contable, el control interno y } \\
\text { el riesgo. }\end{array}$ \\
\hline Docente 3 & Casos prácticos. \\
\hline Docente 4 & $\begin{array}{l}\text { La importancia de aplicar la auditoria se da por la atestación o la fe pública que se han } \\
\text { aplicado los principios de contabilidad. Su necesidad va a depender del objetivo por el cual } \\
\text { se está llevando a cabo. Le enseño las como la auditoria da confiablidad a la información } \\
\text { que se está presentando. Utilizo la discusión de pequeños grupos para identificar cuando es } \\
\text { necesario aplicar auditoria. }\end{array}$ \\
\hline Docente 5 & $\begin{array}{l}\text { Realizar, estudiar, verificar y analizar la entidad contable como una unidad de negocio, bajo } \\
\text { el enfoque sistémico administrativo y contable. Se le aplica a casos prácticos con empresas } \\
\text { que estén dispuestas a colaborar con los estudiantes universitarios. }\end{array}$ \\
\hline
\end{tabular}

Fuente: Instrumento aplicado a los docentes.

Una vez aplicado el instrumento a los docentes, se pudo evidenciar las diversas estrategias que emplean para estimular a los estudiantes a identificar la necesidad de aplicar auditorías, señalando entre ellas la discusión entre pequeños grupos donde se permita reconocer la importancia y aplicabilidad de la auditoría, la revisión, análisis y evaluación de los sistemas de información contable, el control interno y el riesgo, casos prácticos con empresas que estén dispuestas a colaborar 
con los estudiantes universitarios para estudiar, verificar y analizar la entidad como una unidad de negocio, bajo el enfoque sistémico administrativo y contable.

Con base a lo expresado por los docentes, las técnicas que utilizan para impartir estrategias de cuestionamiento relativas a identificar la necesidad de aplicar auditoría, son fundamentales para aquellos estudiantes que aspiren ser auditores, puesto que deben conservar una actitud cuestionante al considerar los factores que indiquen cuando es preciso llevar a cabo una auditoría, bien sea cuando exista un posible fraude o se realice para corregir errores o irregularidades con el fin de optimizar las operaciones de la empresa.

\section{Algunas consideraciones sobre los hallazgos. La triangulación de los datos}

Luego de aplicados los instrumentos de recolección de datos y analizados los resultados desde una perspectiva integral de investigación, abordando aspectos cuantitativos de los hallazgos y cualitativos de los mismos, se muestran a continuación un conjunto de consideraciones finales obtenidas una vez realizada la triangulación de los datos recogidos de los estudiantes, los docentes y los fundamentos teóricos, estas se muestran a continuación.

En relación a la estrategia obtención de evidencia las opiniones presentadas por los estudiantes, indicaron que los docentes le han fomentado estrategias de cuestionamiento para la obtención de evidencia, resaltando que las mismas fueron aplicadas en su mayoría. Para ello, los docentes aplican: el desarrollo de programas de casos y estudios prácticos, la revisión rigurosa y exhaustiva de la información contable tributaria con una perspectiva crítica, el análisis y el estudio de casos, así como, el razonamiento tanto individual como grupal de las evidencias obtenidas.

Ahora bien, dado que algunos estudiantes indicaron en un mínimo porcentaje que no se están aplicando estrategias para la obtención de evidencia, y considerando que un número importante de ellos no opinó al respecto, es fundamental en la función docente con base en los postulados teóricos del Public Company Accounting 
Estrategias profesionales que promueven el escepticismo en los estudiantes de contaduría pública de la ULA-NURR

Oversight Board (2012) la profundización en el estímulo de esta herramienta para promover el escepticismo profesional, tomando en cuenta su requerimiento en el campo laboral y las competencias que aporta al auditor para desarrollar una mentalidad inquisitiva en su actividad profesional independiente.

En este contexto, al triangular los datos obtenidos de los estudiantes, docentes y fundamentos teóricos, se pudo precisar que las estrategias aplicadas para el fomento del escepticismo profesional en lo que respecta a la obtención de evidencia, representan una fortaleza en la formación de los estudiantes de Contaduría Pública de la ULA-NURR, pues brindan instrumentos esenciales para que éste obtenga pruebas que sirvan para determinar la exactitud e integridad de la información, sin embargo, las mismas deben ser profundizadas a fin de mejorar su incentivo en el contexto de los preceptos normativos profesionales.

Por su parte, en lo referente a la aplicación de estrategias tendientes a procedimientos corroborativos los estudiantes, mostraron que durante su proceso de formación universitaria los docentes les han proporcionado estrategias útiles para el implemento de los mismos sobre la información contable, entre las señalan, el uso del juicio crítico para tomar decisiones, corroborar la evidencia obtenida mediante indagaciones sobre la intención que tiene el personal de las entidades y evaluar las respuestas a sus indagaciones corroborándolas con otra información.

Adicionalmente, los docentes indicaron que aplican estrategias como el desarrollo de programas de casos y estudios prácticos, la revisión rigurosa y exhaustiva de la información contable tributaria con una perspectiva crítica, argumentativa e interpretativa considerando que ésta debe ser útil para la toma de decisiones asertivas, así mismo, el análisis de la información con respecto a la correcta aplicación de la NIA en los procedimientos corroborativos y procedimientos de auditoría, estudio de casos prácticos que consistan en consultar, analizar y disertar sobre los riesgos de la auditoría, a fin de permitir la planeación e implementación de respuestas apropiadas a dichos riesgos. 
En efecto, contrastando los hallazgos obtenidos se pudo identificar que las estrategias aplicadas por los docentes para fomentar la mente cuestionante de los estudiantes en el contexto de los procedimientos corroborativos son insuficientes, tomando en cuenta que estos no evalúan las condiciones expuestas en la NIA 620 (IAASB, 2013d) en el apartado A33, por lo cual se recomienda desarrollar sesiones de aprendizaje que promuevan: observación del trabajo de un experto auditor; examen de informes estadísticos de fuentes reputadas y autorizadas; confirmación de personas relevantes con terceros; aplicación de procedimientos analíticos detallados; así como, la re ejecución de cálculos.

En referencia a las opiniones presentadas por los estudiantes, estos señalaron que los docentes le han promovido estrategias para reconocer los indicios de fraude, sobresaliendo entre ellas, el cuestionamiento permanente de la información, el reconocer la posibilidad de que un error importante debido a fraude podría estar presente y la identificación de condiciones que indiquen un incentivo para cometerlo, así pues, los docentes indicaron que emplean la revisión de la normativa, examen de casos y experiencias en el área, la investigación rigurosa y exhaustiva de la información contable y la aplicación de casos prácticos.

En este contexto, los estudiantes manifestaron que la identificación de condiciones que indiquen presiones para cometer fraude, ha sido poco desarrollada, centrándose solo en el conocimiento, análisis y evaluación de la organización bajo el enfoque sistémico, lo cual puede permitir la determinación de los indicios de fraude en las entidades, pero no representa una estrategia significativa de conformidad con lo planteado por Nelson (2009) quien expresa que independientemente de la experiencia profesional con la entidad y su conocimiento, el auditor deberá librarse de creencias sobre la honestidad de la gerencia y su integridad, haciendo fundamental que el docente fomente la inquisición sobre la información financiera que presentan las entidades, así como, sus revelaciones, esto podrá lograrse a través del incentivo de 
Estrategias profesionales que promueven el escepticismo en los estudiantes de contaduría pública de la ULA-NURR

indagaciones externas a la entidad auditada de conformidad con la NIA 240 (IAASB, 2013b).

Así mismo, los hallazgos de la investigación evidenciaron que, durante el proceso de formación profesional de los estudiantes de Contaduría Pública, los docentes les han suministrado estrategias para reconocer la necesidad de aplicar auditoría, sobresaliendo entre ellas, identificarla como una medida de control y para emitir una opinión sobre si los estados financieros han sido preparados en todos los aspectos materiales de conformidad con un marco de información financiera aplicable. Para ello, los docentes emplean; la discusión entre pequeños grupos donde se permita reconocer la importancia y aplicabilidad de la auditoría, los casos prácticos con empresas que estén dispuestas a colaborar con los estudiantes universitarios para estudiar, verificar y analizar la entidad como una unidad de negocio bajo el enfoque sistémico administrativo y contable.

Contradictoriamente, los hallazgos de investigación evidencian que las estrategias de formación profesional aplicadas por los docentes no atienden a cabalidad el precepto normativo establecido en la NIA 200 (IAASB, 2013a) la cual establece que el objetivo de una auditoría es aumentar el grado de confianza de los usuarios en los estados financieros. Considerando, que los aspectos abordados en la formación profesional no han sido desarrollados ampliamente limitando a los estudiantes de Contaduría Pública a la visualización de la auditoría como una actividad profesional enfocada en la revisión, análisis y evaluación de los sistemas de información contable, el control interno y el riesgo, dejando por fuera el aumento de la confiabilidad de los estados financieros, cuya significancia es lo más relevan te en el contexto emergente internacional.

Con base en estas afirmaciones, los resultados de la investigación revelan que las estrategias aplicadas por los docentes para estimular técnicas de cuestionamiento en relación a reconocer la necesidad de aplicar auditoría, deben enfatizar en la aplicación de las Normas Internacionales de Auditoría, despertando la necesidad de 
Yosman Valderrama, Maryelin Trejo, Yoximar Rangel

Telos Vol. 20, No. 2 (2018). 248-276

ampliar las estrategias utilizadas, teniendo en cuenta que el fomento del escepticismo profesional es fundamental para que se lleve a cabo auditorías de calidad.

\section{A modo de conclusión}

En cuanto a expresar una conclusión sobre el objetivo de la investigación, identificar las estrategias de cuestionamiento para formar auditores escépticos, promovidas por parte de los docentes de Contaduría Pública de la ULA-NURR, se pudo conocer las estrategias de formación profesional que los docentes han aplicado sobre sus estudiantes para promover técnicas que permitan cuestionar la información contable, las cuales se precisan a continuación.

En lo que concierne a la obtención de evidencia, los docentes han fomentado la adquisición de información para fundamentar la opinión, el empleo de procedimientos de valoración de riesgo, la aplicación de pruebas de controles y pruebas para detectar errores en los estados financieros, así como, el uso del escepticismo profesional para conseguir suficiente evidencia, para ello aplican el desarrollo de programas de casos y aplicaciones prácticas, la revisión rigurosa y exhaustiva de la información contable tributaria con una perspectiva crítica, el análisis y el estudio de casos, así como el análisis y razonamiento individual y grupal de las evidencias obtenidas.

Con respecto a los procedimientos corroborativos, los docentes han promovido el uso del juicio crítico para tomar decisiones, el corroborar la evidencia obtenida mediante indagaciones sobre la intención que tiene el personal de las entidades y la evaluación de las respuestas a sus indagaciones confirmándolas con otra información, a través de la aplicación de estrategias como, el desarrollo de programas de casos y aplicaciones prácticas, la revisión rigurosa y exhaustiva de la información contable tributaria, el análisis de la información con respecto a la correcta aplicación de la NIA en los procedimientos corroborativos y procedimientos de auditoría y el 
Estrategias profesionales que promueven el escepticismo en los estudiantes de contaduría pública de la ULA-NURR

desarrollo de casos de estudio prácticos que consistan en consultar, analizar y disertar sobre los riesgos de la auditoría.

En relación a los indicios de fraudes, los docentes han impartido el cuestionamiento permanente de la información, el reconocer la posibilidad que un error importante debido a fraude podría estar presente y la identificación de condiciones que indiquen un incentivo para cometerlo, empleando estrategias como, la revisión de la normativa y de casos, al igual que experiencias en el área, la revisión rigurosa y exhaustiva de la información contable y la aplicación de casos prácticos.

En cuanto a, identificar la necesidad de aplicar auditoría los docentes han desarrollado estrategias de formación que incentivan el reconocimiento de aplicar auditorías a los estados financieros como una medida de control que permita emitir una opinión sobre si los estados financieros han sido preparados en todos los aspectos materiales de conformidad con un marco de información financiera aplicable, para ello, se han valido de estrategias como, la discusión entre pequeños grupos donde se permita reconocer la importancia y aplicabilidad de la auditoría, los casos prácticos con empresas que estén dispuestas a colaborar con los estudiantes universitarios para estudiar, verificar y analizar la entidad como una unidad de negocio, bajo el enfoque sistémico administrativo y contable.

En síntesis, se identificar las estrategias de cuestionamiento para formar auditores escépticos, promovidas por parte de los docentes de Contaduría Pública de la ULA-NURR, por lo cual, se pudo concluir que todo profesional contable debe contar con aptitudes fundamentales que le permitan desenvolverse eficientemente en el campo laboral estando capacitado con los conocimientos y valores necesarios para enfrentarse a los hechos o situaciones que observa con una actitud escéptica.

Siendo así, a la universidad le corresponde implementar estrategias de formación que contemplen elementos esenciales de contenido, principios éticos y habilidades que permitan el desarrollo de una actitud inquisitiva en la profesión contable, esta es posible promoverla a través de la aplicación de diversas estrategias 
de enseñanza y pedagogía contable, dentro de las que se pueden mencionar: conversatorios, estudio de casos prácticos, revisión, análisis y evaluación de la información contable, entre otras.

Adicionalmente, la práctica de la enseñanza contable en el ámbito universitario, debe satisfacer los requerimientos establecidos en el Manual de los Pronunciamientos Internacionales de Formación emitido por el Consejo de Normas Internacionales de Formación en Contaduría de la Federación Internacional de Contadores, a través del cual, el órgano regulador presenta una guía de capacitación profesional en contabilidad que puede fortalecer significativa el proceso de formación profesional a los estudiantes de Contaduría Pública de la Universidad de Los Andes, así como, de otras casas de estudio.

\section{Referencias Bibliográficas}

Arias, Fidias (2012). El proyecto de investigación. (6ta edición). Editorial Episteme, C.A. Venezuela.

García, Fernando (2004). El cuestionario: recomendaciones metodológicas para el diseño de cuestionarios. Editorial Limusa. México.

International Auditing and Assurance Standards Board (IAASB) (2016). Handbook of International Quality Control, Auditing, Review, Other Assurance, and Related Services Pronouncements.Editorial International Federation of Accountants. Inglaterra.

Martínez, Francisco; Ramírez, Sergio; Montoya, Javier y Fernández, Ana (2010). La importancia relativa en auditoría: Las nuevas normas internacionales en comparación con la normativa vigente en Iberoamérica. Revista Contaduría y Administración. $\mathrm{N}^{\circ}$ 230, Enero- Abril 2010. España (Pp. 77-103).

Monje, Carlos (2011). Metodología de la investigación cuantitativa y cualitativa. Universidad Surcolombiana. Colombia.

Nelson, Mark (2009).A Model and Literature Review of Professional Skepticism in Auditing.Auditing: a journal of practice \& theory, Vol. 28. $\mathrm{N}^{\circ} 2$. Estados Unidos (Pp. 1-34).

International Auditing and Assurance Standards Board (2013a). Norma Internacional de Auditoría $\mathrm{N}^{\circ}$ 200. Objetivos globales del auditor independiente y realización de la auditoría de conformidad con las normas internacionales de auditoría. Instituto Mexicano de contadores. Inglaterra.

International Auditing and Assurance Standards Board (2013b). Norma Internacional de Auditoría $\mathrm{N}^{\circ}$ 240. Responsabilidad del auditor en la auditoría de estados 
Estrategias profesionales que promueven el escepticismo en los estudiantes de contaduría pública de la ULA-NURR

financieros con respecto al fraude. Instituto Mexicano de contadores. Inglaterra.

International Auditing and Assurance Standards Board (2013c). Norma Internacional de Auditoría $\mathrm{N}^{\circ}$ 500. Evidencia de Auditoría. Instituto Mexicano de contadores. Inglaterra.

International Auditing and Assurance Standards Board (2013d). Norma Internacional de Auditoría $\mathrm{N}^{\circ}$ 620. Utilización del Trabajo de un Experto Auditor. Instituto Mexicano de contadores. Inglaterra.

Public Company Accounting Oversight Board (PCAOB). (2012). Maintaining and applying professional skepticism in audits. [Documento en Línea]. Extraído de: $\quad$ https://pcaobus.org/Standards/QandA/12-04-2012_SAPA_10.pdf. Consulta: 01/09/2016.

Ramírez, Tulio (1999). Como hacer un proyecto de investigación. (1era ed.). Editorial Panapo. Venezuela.

Rodríguez, Ernesto (2005). Metodología de la investigación. (1era ed.). Universidad Juárez Autónoma de Tabasco. México.

Scribano, Adrian (2007). El proceso de investigación social cualitativo. Editorial Prometeo Libros. Argentina.

Viloria, Norka (2013). El Escepticismo Profesional: Estrategias de aula para estudiantes de Contaduría Pública. Revista Actualidad Contable. $N^{\circ} 27$, Julio-Diciembre de 2013. Venezuela (Pp. 102-119). 